\title{
Energy efficient WSN using hybrid modification PEGASIS with ant lion optimization
}

\author{
Ahmed Abdul Azeez Asmael, Basman Al-Nedawe \\ Middle Technical University, Technical Institute of Baquba, Baquba, Dayala, Iraq
}

\begin{tabular}{l} 
Article Info \\
\hline Article history: \\
Received Feb 20, 2021 \\
Revised Jun 7, 2021 \\
Accepted Jun 18, 2021 \\
\hline
\end{tabular}

\section{Keywords:}

Ant lion optimization

Energy consumption

Nodes

PEGASIS

Wireless sensor

\begin{abstract}
Wireless sensor nodes consist of tiny electronic devices that can sense, transmit, and measure data from physical environments such as the field of minter surveillance. These sensor nodes significantly depend on batteries to gain energy which is used to operations associated with communication and computation. Generally, designing communication protocols is feasible to achieve effective usage of these energy resources of the sensor node. Both reported medium access control and routing can achieve energy-saving that supporting real time functionality. This paper emphasizes the use of hybrid modified PEGASIS-Ant lion optimization. Several steps are entailed in this research. First is random distribution of node followed by clustering the map as a circular region. Then, the nodes are connected to the closest node in that region. In consequence, PEGASIS-Ant lion optimization is applied to enhance the connection of the nodes and accomplish the maximum life batter of the sensor. At last, the experiments performed in this work demonstrate that the proposed optimization technique operates well in terms of network latency, power duration and energy's consumption. Furthermore, the life span of the nodes has improved greatly by $87 \%$ over the original algorithm that accomplished a rate of life nodes of $60 \%$.
\end{abstract}

This is an open access article under the CC BY-SA license.

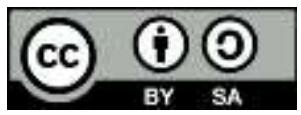

\section{Corresponding Author:}

Basman Al-Nedawe

Department of computer system

Middle Technical University

Email: b.al-nedawe@mtu.edu.iq

\section{INTRODUCTION}

Advancements and innovations occurring in the field of wireless communication play a key role in developing wireless sensor networks that consist of minor devices that can gather information by working cooperatively [1]-[3]. These tiny sensing devices are known as nodes and contain the following components: (1) central processing unit, utilized to process data, (2) memory, utilized to store data, (3) battery, utilized to obtain energy, (4) transceiver, utilized to receive and send signals amongst various nodes. It is important to realize that different nodes have different sizes depending on the function it carries out. For instance, in applications related with military or surveillance aspects, the size of the sensor node may be invisible to the naked eye. In terms of cost, factors like memory space available for data storage, battery and the speed required to process data (information) all influence massively [4], [5]. Nowadays, there are several applications of WSNs in various fields, which can all be employed, however are not limited. For examples, areas of trade and industry such as healthcare benefit by monitoring environment and habitat, or surveillance (like military fields). The use of WSNs is witnessing a noticeable proliferation and at the same time their use is hampered by an issue of energy-related constraints regarding the battery's limited duration [6], [7]. 
Since each node is energy-dependent in its activities, this operation poses a challenge in WSNs; where the dysfunction of any node interrupts the whole system overall. Typically, each node can take a number of patterns. At some points, it might be an active pattern (mode) (in terms of receiving and transmitting data/ information), or an idle pattern or a sleep pattern. Nodes are seen to be in active mode whilst receiving or sending data. The following points are suggested to save energy or reduce energy consumption produced by communication processes in WSNs [8], [9],

a) Scheduling the mode of the node, i.e. receiving mode, transmitting mode, sleep, or idle mode (pattern).

b) Altering the range of transmission among sensing nodes.

c) Employing well-organized whilst ensuring highly efficient routing and data-gathering methods.

d) Prevent and avoid dealing with any undesirable data.

Practically, battery is regarded as the sole source for life sustainment of nodes in WSNs. This is because any communication taking place with other nodes or any sensing activities would need great amounts of energy for both processing and transmitting the gathered data to the base station (BN). In most cases such as surveillance applications, replacing battery that depletes or drains energy is highly suggested. Therefore, a lot of researchers were having multiple attempts at discovering suitable energy-efficient protocols for WSNs. The aim behind such action is to eliminate issues such as those mentioned above [10]-[12].

It is said that a protocol has real-time support as long as its speed and reliability towards reactions in the network are found in their upmost extent. Moreover, a protocol is expected to provide redundant data to BS by using the gathered data from the whole sensing node. Appropriately, any delay found in the network whilst transferring data from sensor nodes to base station should be short. Thus, the result is a rapid reaction [13], [14]. The work accomplished in this paper makes use of hybrid ant lion optimization as well as modified PEGASIS. The objectives of this work are to improve the nodes sensors network in terms of network latency, power duration and energy's consumption. This will be achieved using hybrid modified PEGASIS-Ant lion optimization. Several steps will be entailed in this research. First, a random distribution of node followed by clustering the map as a circular region. Then, the nodes are connected to the closest node in that region. Consequently, PEGASIS-Ant lion optimization will be applied to enhance the connection of the nodes and accomplish the maximum life batter of the sensor.

\section{RELATED WORK AND BACKGROUND THORY}

\subsection{Literature survey}

In the last ten years, researchers have conducted a lot of studies concerning WSN energy consumption issues where a survey of leading approaches and protocols with the main aspects of its comparison are provided,

a) Rina Mahakud et al. in 2016 [15], proposed PEGASIS a near optimal chain-based protocol, which was used for extending network lifetime. In PEGASIS, each node can communicate with only a neighboring node, performs a chain, and elects a leader from the chain. This would collect any data from the surrounding nodes which are sent to the BS. As a result, it is possible to achieve a reduced power consumption that can be utilized to increase both network's competence and lifetime.

b) Doko Bandur Đoko Banđur, Branimir Jakšić, Miloš Banđur, and Srđan Jović. in 2019 [16], focused on analysis linked to energy efficiency in (WSNs) that is employed in the field of smart agriculture, environment inputs and requirements, analysis and planning, and design phases. Simulation was by deploying 500 sensor nodes on in area of 800 width by 800 length $\mathrm{m}^{2}$. The average of energy consumption was found to be $49.29 \%$.

c) Raj Priyadarshini, in 2019 [17] presented work that applied a hybrid mechanism for improving energy efficiency to obtain faster transmission of data in an underwater WSN. Therefore, antcolony optimization (ACO) routing with markov-chain monte-carlo (MCMC) algorithm were applied. This is a method used to handle this trouble and to capturing any transmission loss in the Markov Chain Monte Carlo Method. More specifically, channel status information forecast prediction algorithm was used. In particular, the process of evaluation of experimental simulations was carried out by using performance evaluation.

d) Somauroo and V. Bassooin 2019 [18], illustrated routing algorithms that increase the sensor nodes' lifetime in 3D area wireless sensor networks. This was accomplished by using PEGASIS protocol and genetic algorithm to establish the full chain. The protocol was executed for networks considering separate cases of a fixed BS both outside and inside the network. The results showed a significant enhancement in the lifetime of PEGASIS by $81.7 \%$.

e) K. Karunanithy, B. Velusamy in 2020 [19], demonstrated a clustertree based energy efficient data gathering (CTEEDG) protocol to lengthen both throughput and lifetime of the WSN. In the phase of inter-cluster communication, tree topology is formed amongst clusters towards the (BS) that guarantees 
the obtainability of the congestion-free shortest path to the base station. From the simulation results, CTEEDG has performed better than the DL-LEACH by $38.28 \%$ where FAMACROW was better by $28.81 \%$ in the throughput.

\subsection{PEGASIS (power efficient gathering in sensor information systems)}

The main concept behind PEGASIS is that it uses all nodes to transmit or receive data with the nearest neighboring nodes. A chain is formed in order to achieve this, as displayed in Figure 1. All the nodes that are responsible for gathering data, combine with data from the neighboring nodes where overall are sent to the nearest neighboring node. Specifically, this method ensures that all nodes receive and fuse their data, where the data is later passed to the next neighbor in the form of a chain until all data arrives to BS. It is acknowledged that each node in the network rotates as the chain leader and is responsible for transferring fully integrated data that is obtained by the node chain to the BS [20].

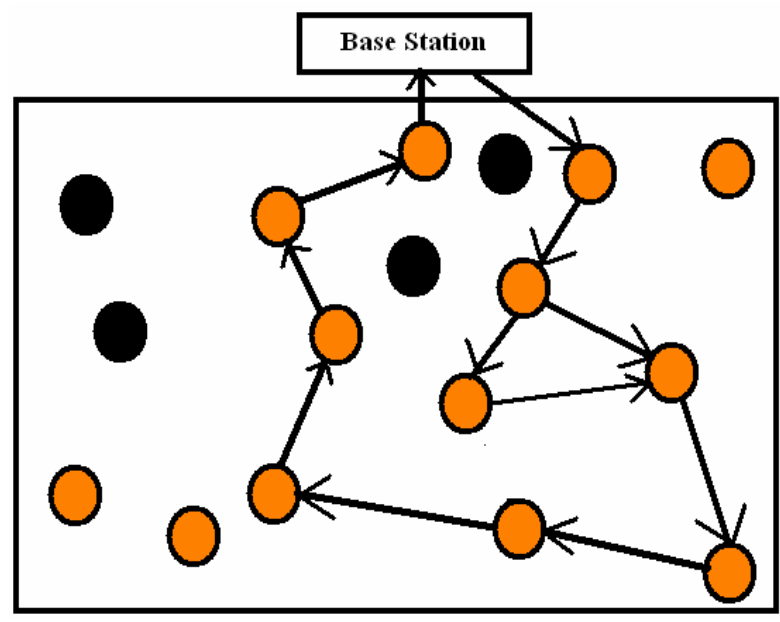

Figure 1. Formation of chain using nodes in PEGASIS

Using this approach, energy load is equally dispended between sensing nodes in the network where the whole network nodes is used to form the chain and perform uncomplicated data- forwarding operations. If a node dies in the produced chain, a new chain is made where the dead nodes are removed [21].

\subsection{Radio and energy model}

Demonstrated in Figure 2 is the first-order radio model which is used for computing energy consumption associated with communicating nodes. This stage is simple to be applied and exploited mostly in literature of wireless sensor networks [22]. Hence, it becomes easier and more reliable to make comparisons with previous protocols. It is a fact that energy is an essential factor for running transmitter and receiver circuits. The latter is a function of the number of bits (k) only [23] as presented in (1).

$$
\mathrm{E}_{\frac{\mathrm{Tx}}{\mathrm{Rx}}-\mathrm{elec}}=\mathrm{E}_{\text {elec }} * \mathrm{k}
$$

Where $E_{\text {elec }}$ is the energy used in each bit to operate a transmitter or a receiver. Transmission power that is used by the amplifiers represents a function of both distance of transmission and the number of bits.

$$
\mathrm{E}_{\mathrm{Tx}-\mathrm{amp}}\left\{\begin{array}{c}
\epsilon_{\mathrm{fs}} * \mathrm{k} * \mathrm{~d}^{2}, \text { if } \mathrm{d}<\mathrm{d}_{0} \\
\epsilon_{\mathrm{mp}} * \mathrm{k} * \mathrm{~d}^{4}, \text { if } \mathrm{d}<\mathrm{d}_{0}
\end{array}\right.
$$

$d_{0}$ is a threshold that is utilized for determining multipath, free space and is accomplished by,

$$
\mathrm{d}_{0}=\sqrt{\frac{\epsilon_{\mathrm{fs}}}{\epsilon_{\mathrm{mp}}}}
$$


where the constants $\in$ fs and $\in \mathrm{mp}$ are employed in the multipath model and free space independently. Thus, the consumption energy required for transmitting packets is,

$$
\mathrm{E}_{\mathrm{Tx}}=\left\{\begin{array}{c}
\mathrm{E}_{\text {elec }} \times \mathrm{k}+\epsilon_{\mathrm{fs}} \times \mathrm{k}+\mathrm{d}^{2}, \text { if } \mathrm{d}<\mathrm{d}_{0} \\
\mathrm{E}_{\text {elec }} \times \mathrm{k}+\epsilon_{\mathrm{mp}} \times \mathrm{k}+\mathrm{d}^{4}, \text { if } \mathrm{d}<\mathrm{d}_{0}
\end{array} .\right.
$$

the energy that is essential for the process of reception consists of the energy needed to operate the circuit only [24].

$$
\mathrm{E}_{\mathrm{Rx}=} \mathrm{E}_{\mathrm{elec}} \times \mathrm{L} \ldots \ldots
$$

The cost of energy for data aggregating is,

$$
\mathrm{E}_{\mathrm{DA}-\mathrm{tot}}=\mathrm{S} \times \mathrm{k} \times \mathrm{E}_{\mathrm{da}} \cdots \cdots
$$

where s represents the signal number that is gathered and $\mathrm{E}(\mathrm{da})$ represents the energy for each bit spent in the process of aggregating [22].

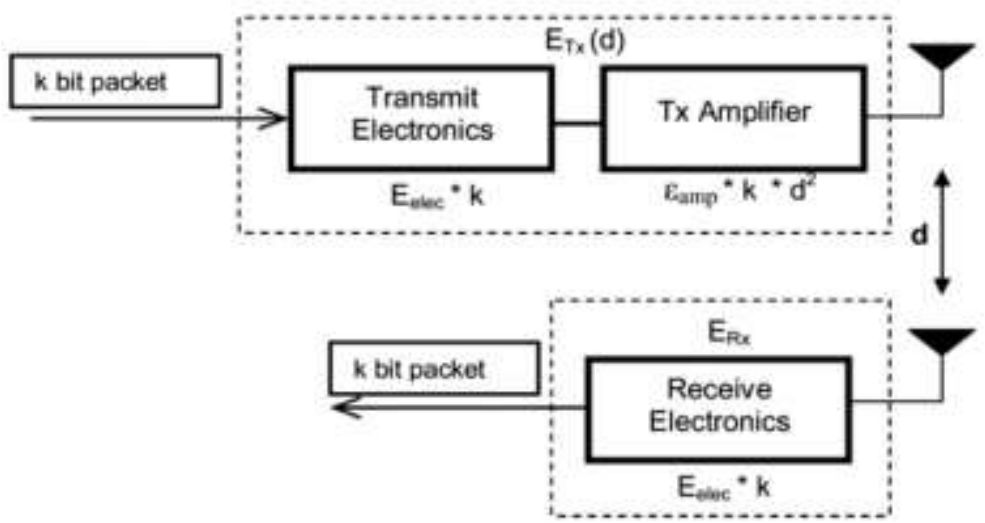

Figure 2. First-order radio model [20]

\subsection{Ant lion optimizer (ALO)}

The ALO is an algorithm that is able to determine the most suitable designs for a major portion of the existing traditional engineering issues. This idea points out that this type of algorithm has various advantages in finding solutions to constrained problems in many research fields. The ALO algorithm's core inspiration is initially from the foraging behavior of ant-lion's larvae. Considerably, the cone edge is satisfactorily sharp for insects to be trapped without any difficulty. It is known that Ant Lion Optimizer algorithm encourages interactions to take place between ants and ant lions in a snare. For this interaction to happen, ants are required to walk in the search space where antlions are allowed to chase them and make use of the most suitable snares. For computation purposes, the random movement of ant is carefully chosen for modeling ants' movements. This is further understood [25],

$$
\begin{aligned}
& X(t)=\left[0, \operatorname{cumsum}\left(2 r\left(t_{1}-1\right), \ldots, \operatorname{cumsum}\left(2 r\left(t_{n}-1\right)\right)\right.\right. \\
& r(t)=\left\{\begin{array}{c}
1 \text { if rand }>0.5 \\
0 \text { if rand }<0
\end{array}\right.
\end{aligned}
$$

where $t$ indicates the steps of random walk and rand represents a random number created in interval of $[0,1]$. The behaviour noticed by Antlions' hunt shows that the random walk used may possibly vary around the source (red curve). It may either have a descending behavior (a blue curve), or an increasing trend (a black curve). In consequence, the ants' position is noted and applied through optimization in the next matrix [23], 


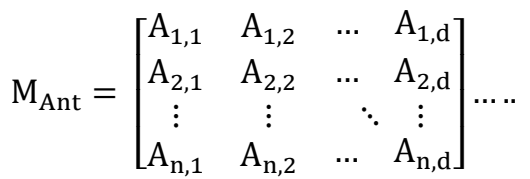

where $M(A n t)$ is the matrix to record the fitness of each ant, $A(i, j)$ represents the value of the $j$-th dimension of the $\mathrm{i}$-th ant; $\mathrm{f}$ demonstrates the objective function and finally $\mathrm{n}$ represents the number of ants. It is suggested to keep in mind that possibly ants may hide somewhere in the search space. To maintain their locations and fitness-values, these matrices are used,

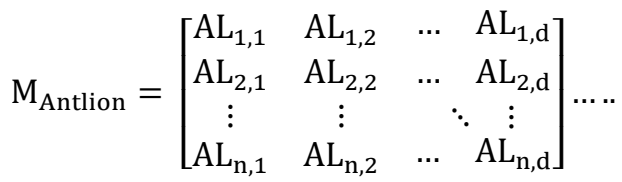

where $\mathrm{M}($ Ant-lion $)$ is the matrix to keep each ant-lion's post, AL(i,j) indicates the $\mathrm{j}$-th dimension's value of $\mathrm{i}$ th antlion, $d$ is the number of variables (dimension) and lastly but not least is $n$ which represents the number of antlions.

$$
M_{\mathrm{OA}}=\left[\begin{array}{c}
\mathrm{f}\left(\left[\mathrm{AL}_{1,1} \mathrm{AL}_{1,2} \mathrm{AL}_{1, \mathrm{~d}}\right]\right) \\
\mathrm{f}\left(\left[\mathrm{AL}_{2,1} \mathrm{AL}_{2,2} \mathrm{AL}_{2, \mathrm{~d}}\right]\right) \\
\vdots \\
\mathrm{f}\left(\left[\mathrm{AL}_{\mathrm{n}, 1} \mathrm{AL}_{\mathrm{n}, 2} \mathrm{AL}_{\mathrm{n}, \mathrm{d}}\right]\right)
\end{array}\right] \ldots \cdot
$$

$\mathrm{M}(\mathrm{OA})$ is the $2 \mathrm{D}$ array that maintains the fitness of every antlion, AL(i,j) highlights the $\mathrm{j}$-th dimension's value of $\mathrm{i}$-th antlion, f depicts the objective function and finally $\mathrm{n}$ represents the number of antlions. During the process of optimization, all Random walks are dependent on (7). The idea behind such process is that ants work by updating their locations with random walk at each step of optimization. Since each search space has a boundary, nevertheless, (7) cannot be used immediately to update the ants' position. Moreover, to maintain random walks inside the space of search, normalization takes place where (12) is used (min-max normalization) [23],

$$
X_{i}^{t}=\frac{\left(X_{i}^{t}-a_{i}\right) \times\left(d_{i}-c_{i}^{t}\right)}{\left(d_{i}^{t}-a_{i}\right)} \ldots \ldots
$$

where a(i) portrays the minimum random walk, $d_{i}^{t}$ represents the maximum of (i)-th variable at t-th iteration and lastly $c_{i}^{t}$ represents the minimum of whole variables at $(\mathrm{t})$-th iteration. Each iteration is expected to employ (13) to guarantee that random walks are performed inside the space of search. From what is comprehended, antlions' traps have an influence on random walks of ants. Thus, to model this assumption, a mathematical method is used as both (13) and (14) propose,

$$
\begin{aligned}
& c_{i}^{t}=\text { Antlion }_{i}^{t}+c^{t} \ldots \cdot \cdot \\
& d_{i}^{t}=\text { Antlion }_{j}^{t}+d^{t} \ldots . .
\end{aligned}
$$

both (14) and (15) demonstrate that ants arbitrarily walk in a hyper sphere resolved by the vectors $\mathrm{c}$ and $\mathrm{d}$ around a selected ant lion. Figure 3 illustrates a theoretical model linked to this behavior where it displays a search space consisting of two dimensions. It is noted that ants are required to transfer inside a hyper sphere around the selected ant lion. Using the suggested mechanisms, the pants can form traps proper to their fitness level. Moreover, ants are required to move around randomly. On the other hand, ants release sands out of the hole center as soon as they realize that an ant is trapped in the snare. This form of action drops the stuck ant that is attempting to run away. To model such behavior regarding mathematics, the random walking radius of the ants decreases, in an adaptive manner. These (15) and (16) were provided in this aspect,

$$
\begin{aligned}
c^{\mathrm{t}} & =\frac{\mathrm{c}^{\mathrm{t}}}{\mathrm{I}} \ldots \ldots \\
\mathrm{c}^{\mathrm{t}} & =\frac{\mathrm{d}^{\mathrm{t}}}{\mathrm{I}} \ldots \ldots
\end{aligned}
$$




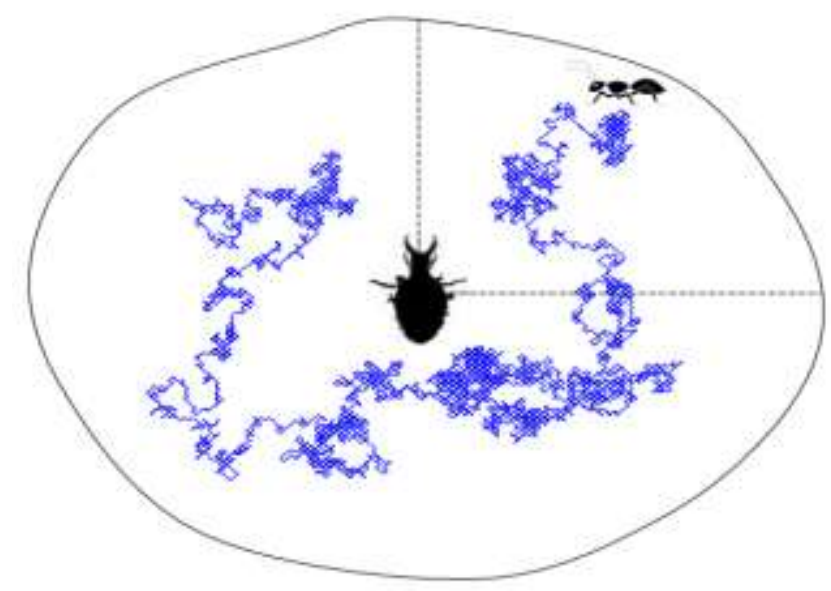

Figure 3. An ant randomly walking inside an antlion's trap

The last phase of hunting takes place when an ant arrives at the bottom of the hole and is stuck in an antlion's jaw. Following this, the ant can drag the ant into the sand and ingest its body fully. For encouraging such procedure, prey-catching is assumed to occur when ants get more competent to go into the sand than their opponent. After that, the antlion is expected to alter its position to the latest ant-hunting place to improve its opportunity to hunt a new prey. In terms of what was discussed, (17) is applied,

$$
\text { Antlion }_{\mathrm{j}}^{\mathrm{t}}=\text { Ant }_{\mathrm{i}}^{\mathrm{t}} \text { if } \mathrm{f}\left(\mathrm{Ant}_{\mathrm{i}}^{\mathrm{t}}\right)>\mathrm{f}\left(\text { Antlion }_{\mathrm{j}}^{\mathrm{t}}\right) \ldots .
$$

where $t$ represents the existing iteration, ant $\mathrm{i}$ highlights the spot of $\mathrm{i}$-th ant at $\mathrm{t}$-th iteration and antlion $\mathrm{j}$ refers to the site of the selected $\mathrm{j}$-th antlion at $\mathrm{t}$-th iteration.

The term Elitism is regarded as a major feature from evolutionary algorithms that has the ability to provide best solutions for any stage at the process of optimization. In this study, the finest antlions were stored in each iteration and named as elites. Considerably, ensuring that the elite is the fittest antlion, it can pose an impact on ants' movement overall by iteration. Hence, an assumption is indicating which states that each ant moves randomly around a specified antlion by the roulette wheel and the elite simultaneously. This is shown in (18),

$$
\operatorname{Ant}_{\mathrm{i}}^{\mathrm{t}}=\frac{\mathrm{R}_{\mathrm{A}}^{\mathrm{t}}+\mathrm{R}_{\mathrm{E}}^{\mathrm{t}}}{2} \ldots \ldots
$$

where $\mathrm{R}(\mathrm{t}, \mathrm{A})$ is the random movement around the antlion selected by the roulette wheel at $\mathrm{t}$-th iteration, $\mathrm{R}$ $(\mathrm{t}, \mathrm{E})$ points out the random movement around the elite at $\mathrm{t}$-th iteration, and lastly Ant $(\mathrm{t}, \mathrm{i})$ refers to the spot of i-th ant at t-th iteration.

\section{METHODOLOGY}

One of the most essential aspects posing an impact on high-quality clustering is the simultaneous consideration of the two criteria consisting of the distances within a cluster (inter-cluster) and the distances within two or more clusters (intra-cluster). Most recommended methods indicate that only one of these criteria is considered or even none have. When examining the methods at which these criteria are perceived, both quality and precision of the cluster are not computed at the end stage of clustering. Certainly, error rate in cluster production is regarded as one of the most significant problems impacting high-quality clustering. In this work, some recommendations are given to be able to measure the quality of the cluster. These criteria are listed,

a) The first criterion depends on the densities within a sole cluster amongst two or more clusters. In the case where the criterion is small, the cluster's quality is considered better.

b) The second criterion points out an error taking place during clustering. The action of arranging nodes in a cluster leads to the high-quality clustering criteria. To further understand the idea, if more regular nodes cover the surface of the cluster, the cluster will be balanced greatly, and nodes' consumption of energy will decrease. 
c) The third criterion emphasizes the nodes' balance within the clusters. The precision range linked to this criterion is established according to both measurement error and statistical assumptions.

As the criteria is overviewed and comprehended, the clustering process is explained step-by-step,

a) The first step: $\mathrm{N}$ sensor nodes spread randomly (non-deterministic deployment) in a square field with a length of $\mathrm{M}$ with a static BS.

b) The second step: Every node has a constant amount of energy Eo.

c) The third step: Once the nodes are spread, the map is clustered into circular regions ensuring they are around the base station. Typically, the first region would contain all nodes with less than $20 \mathrm{~m}$ from the base station. Then, the second region would contain any node that is in the range of $21-40 \mathrm{~m}$ from the base station. Lastly, the third region would contain all nodes having $41-60 \mathrm{~m}$ from the base station. This pattern is continued.

d) The fourth step: Once the cluster map is completed, all nodes are linked in between where each node connects to its neighboring node in its region. For example, a node found in the second region would connect to its nearest node in the first region. The last step would be employing the Ant lion optimization on the modified PEGASIS. Overall, this would justify the connection of nodes and improves lifetime of the nodes.

Summary for the proposed node

a) All sensing nodes are positioned randomly and continue being static even after being located.

b) Each sensor node possesses a matchless ID to be distinguished from other nodes.

c) It cannot replace the battery of all the sensing nodes that possess the same primary energy.

d) The map is clustered into circular regions around the base station (BS).

e) Each node is connected to its most neighboring node within the area that is preceded.

f) The Ant lion optimization is applied on the modified PEGASIS where nodes are successfully interlinked, and their lifetime is massively developed.

g) It is possible to adjust transmission power of sensors depending on communication distance.

\title{
3.1. Ant lion optimization (ALO)
}

Once the model of modified PEGASIS is prepared successfully, the ant lion is applied as can be seen in algorithm (1). To develop the lifetime of energy, any operators recommended in the previous subsections were used. Therefore, it is possible not to define the ALO optimization algorithm. However, the ALO algorithm is considered as a function having three tuples functions that estimate worldwide optimization issues. This is outlined further,

\author{
Comprehending ALO algorithm: \\ MPEGASIS refers to the start population \\ Measure the fitness of ant lions \\ Choose the most suitable ant lion (assume as the elite) \\ While the end criterion in unassured yet \\ For each ant lion \\ Employ the Roulette wheel for the selected ant lion \\ Update parameters c and d in accordance with (16) (17). \\ Generate an unarranged walk then normalize it in accordance with (7) and (13). \\ Inform the new location of the ant line \\ End loop for \\ Compute all ant's lion fitness \\ Exchange an antlion with its correlate ant turn into fitter (18) \\ Upgrade the elite in the case when an antlion is fitter than the elite \\ End while \\ Return elite
}

\section{RESULTS AND DISCUSSION}

Important steps to be taken are to both test and evaluate the presented work in this study. This is in terms of energy consumption in PEGASIS protocol. All tests linked to the performed experiments and simulations were carried out using Matlab 2019. As a remind, this proposed work focused on hybrid ant lion algorithm with modified PEGASIS. The procedure began by determining the map size $\left(200 * 200 \mathrm{~m}^{2}\right)$ followed by random distribution of nodes around the base station. The position was 100,100 as noticed in Figure 4. For the parameters of this work, Table 1 can be used.

To cluster the map into circular regions, computing the Euclidean distance between the nodes and the $\mathrm{BS}$ is required to be carried out. Afterwards, the circular regions are divided where the first region contains all nodes within a distance of less than $20 \mathrm{~m}$ from the BS, the second region contains all nodes within 
a distance of 20 to $40 \mathrm{~m}$ from the BS and so on the rest of the regions. Figure 5 clearly shows this aspect. Subsequently, linking the nodes occurs where various nodes between regions were interlinked. This is where each node was connected to its nearest node in the area that preceded it. This can be seen certainly in Figure 6. Once nodes are linked, the ant lion optimization is employed to reduce energy consumption via justifying the link node. Figure 7 proves that this concept to be correct.

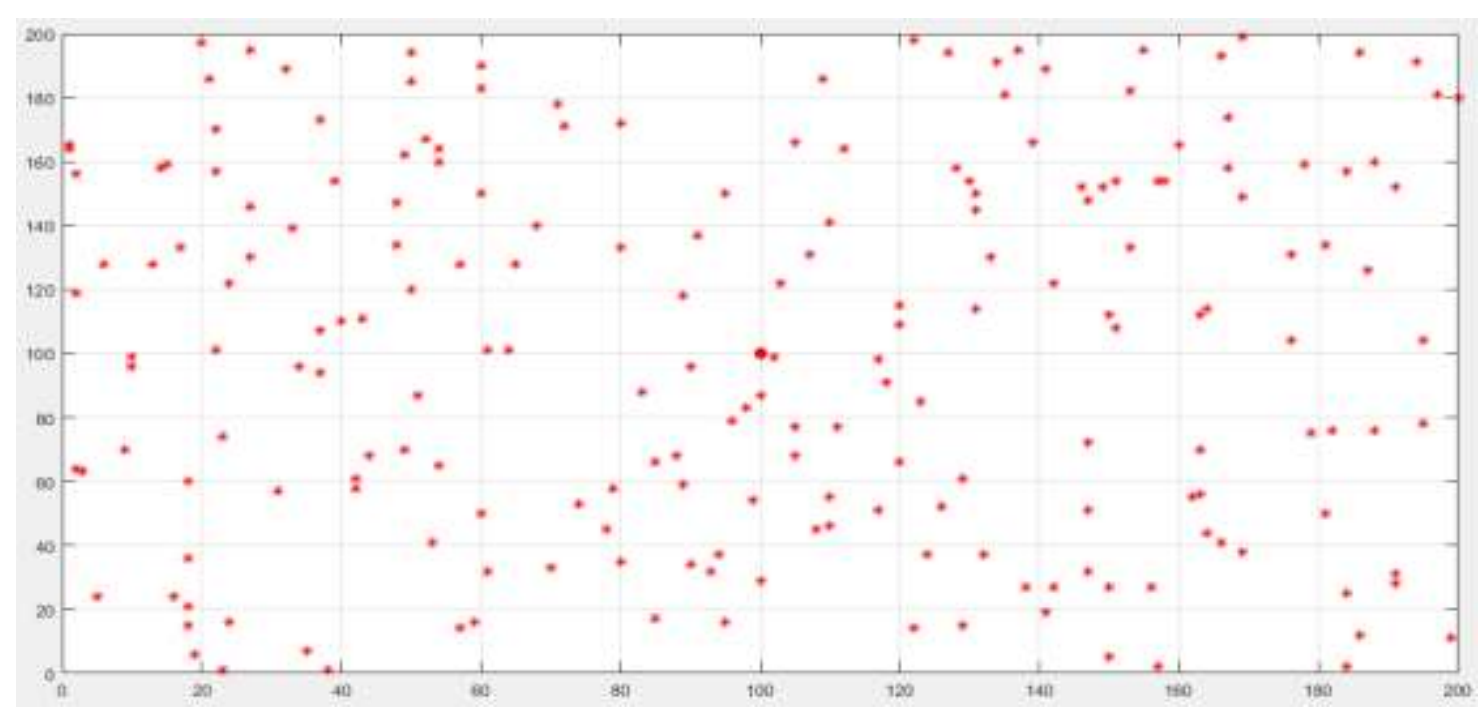

Figure 4. Random distribution of nodes

Table 1. WSN simulation parameters for the proposed protocols

\begin{tabular}{cc}
\hline Parameters & Values \\
\hline The Area & $200 * 200 \mathrm{~m}^{2}$ \\
Initial energy & 0.003 \\
Number of nodes & 200 \\
Bit_rate & $1 \mathrm{Mb} / \mathrm{sec}$ \\
Packet Length & $500($ Byte) \\
Media Access Control Layer & IEEE 802.11 \\
\hline
\end{tabular}

Note: Packet size represents the amount of data that collected from the sensors

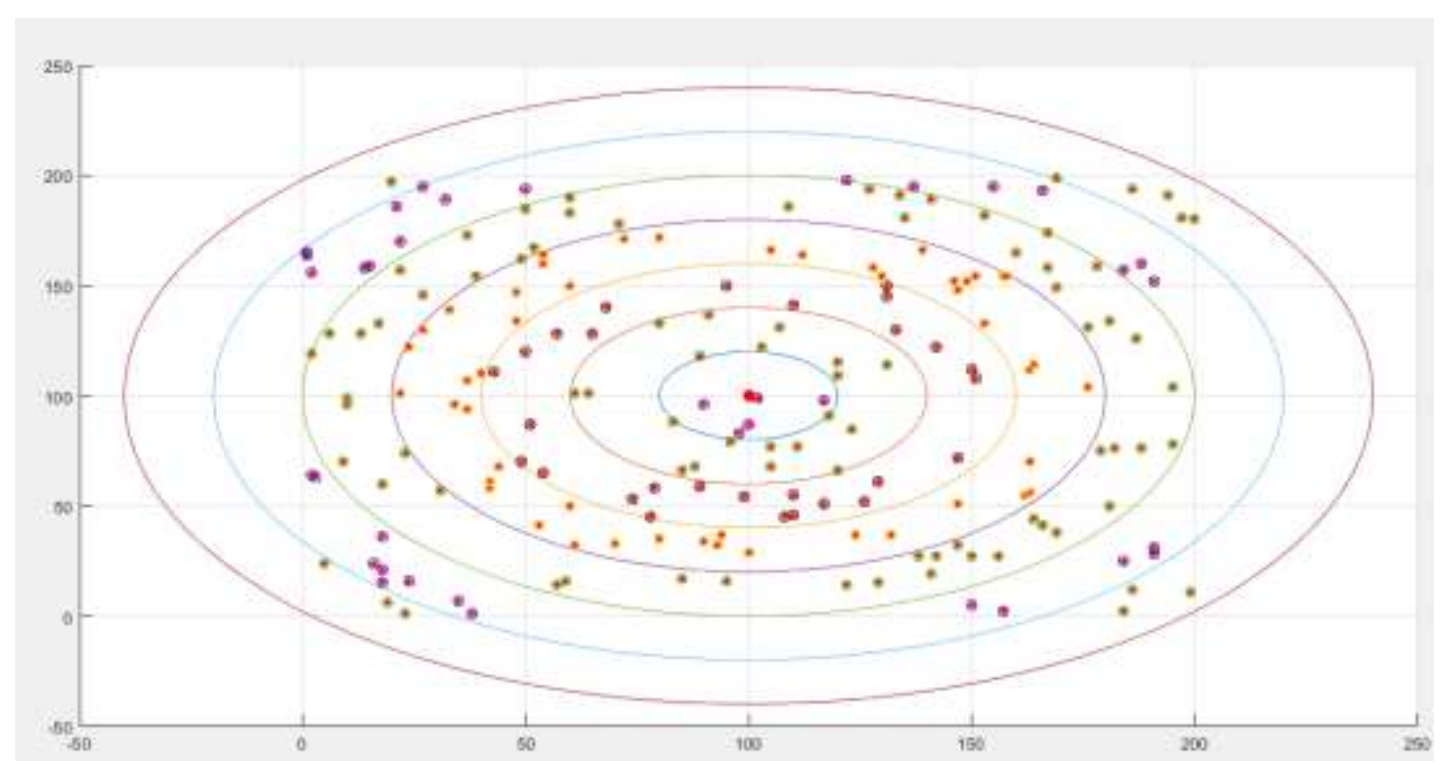

Figure 5. Clustering of the map into circular regions 


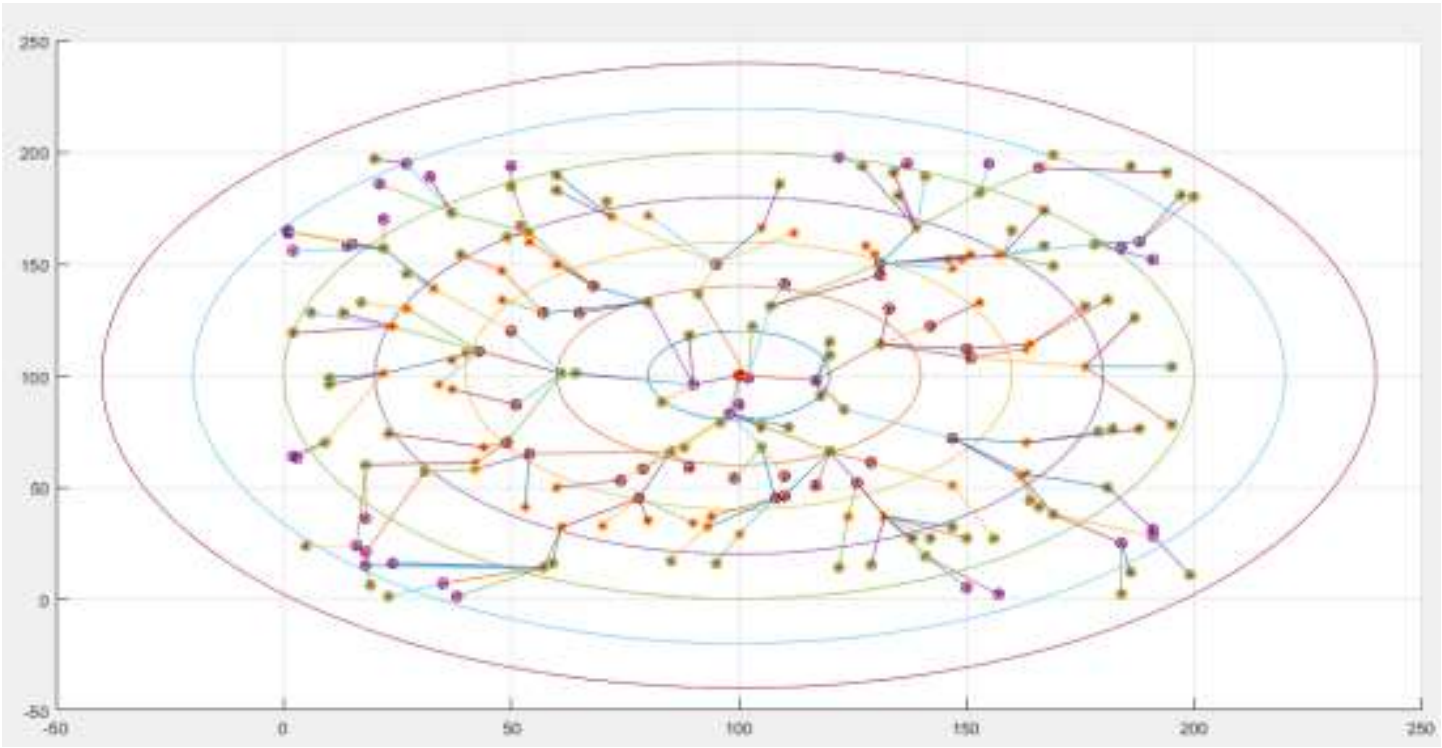

Figure 6. Linking nodes with other nodes

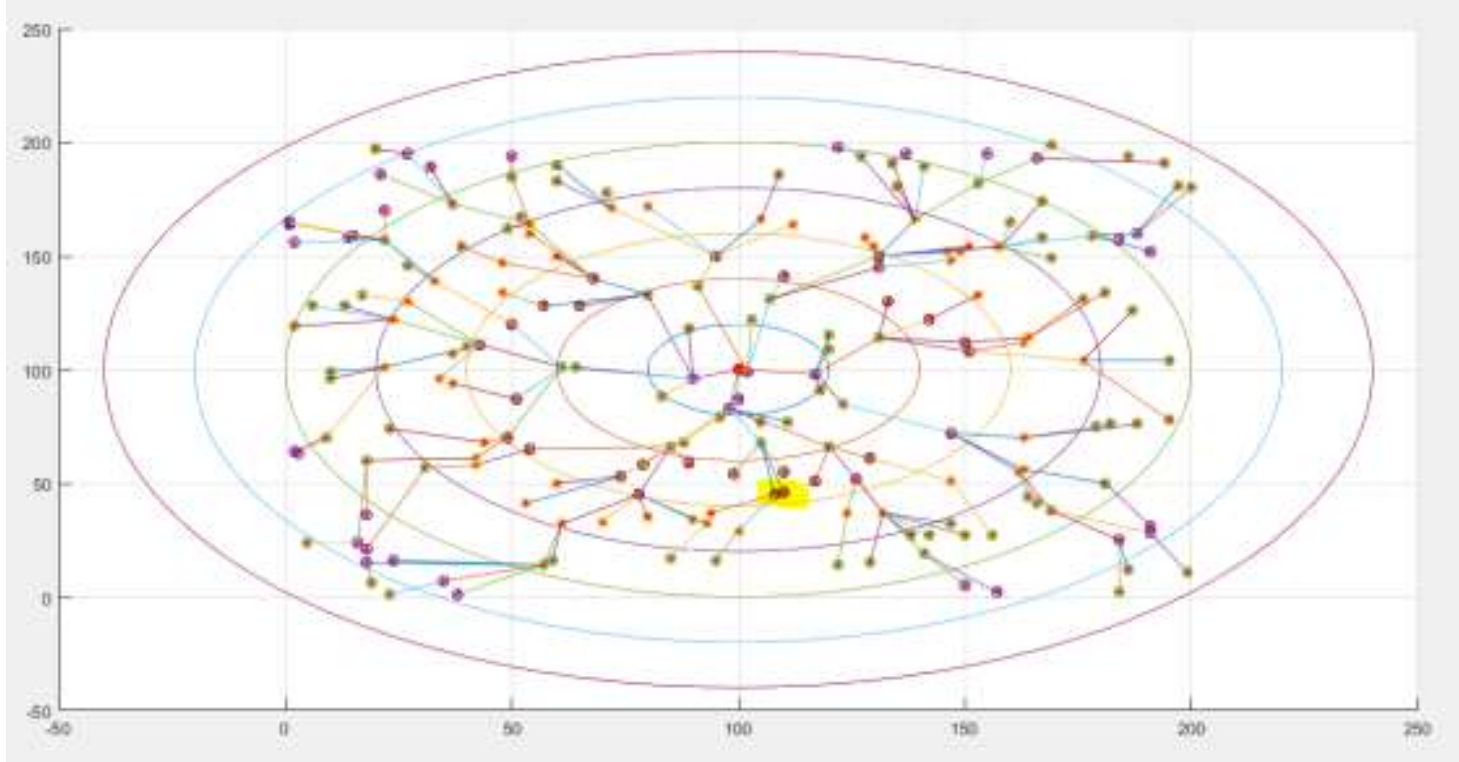

Figure 7. Application of ALO in hybrid MPEGASIS

\section{PERFORMANCE METRICS}

The reason behind using performance metrics is to analyse and test networks according to specific criteria to justify the simulated scenarios' behavior. In particular, when performance metrics are used, the improvement level of the presented protocols in comparison to other ones is illustrated. Any of the clustering protocols that aim to review and evaluating tests of most WSNs highlight the importance of evaluating HND (half nodes die) and FND (first node die) in the network. Besides, the average dissipated energy would be also included. It is agreed on that no sensor activity within the network is perceived. The stability period in the clustering protocol is verified for following:

a) First Node to Die: The time that the $1^{\text {st }} \mathrm{t}$ dead node appears.

b) Half Nodes to Die: The time between network operation starting to the time of half nodes being dead.

As the network lifetime of the proposed protocol was confirmed in comparison to other ones, a number of performance measures, comprising average energy consumption, productivity, packet delivery ratio and end-to-end delay, can be discovered, 
1) Average Throughput: Throughput is used to measure data packets that are received successfully, per unit time via (19).

Avg. throughput $=(\mathrm{z} /$ total time $) \ldots .$.

Where $\mathrm{z}$ represents the net of successfully received packets.

2) End to End Delay: Refers to the time taken between reception and transmission of data packets. It can be calculated from (20).

Average end to end delay $=\sum_{i=1}^{m} \frac{\left(T_{i}^{r}-T_{i}^{S}\right)}{m} \ldots$

Where $\left(T_{i}^{r}-T_{i}^{S}\right)$ indicates the variation in reception and transmission time of the packet and $\mathrm{m}$ in the total number of transmitted packets.

3) Average energy consumed: Energy consumption happens in transmitting, receiving, and processing of the packet by each node through the operation of the network. Energy consumption in each node (i) is worked out from this formula:

$\mathrm{Eci}=\mathrm{ET}+\mathrm{EPi}+\mathrm{Eri}$

Where Eci points out the total consumed energy, ET refers to the consumed energy through the process of transmitting, EP is the consumed energy through processing of packet, $E R$ is the consumed energy through the process of reception and finally (i) is viewed as quantity of the nodes. Finding out the average dissipated energy also means finding the percentage of the total network energy-consumption to the (n) nodes.

$$
\text { Average Energy Consumption }=\sum_{i=1}^{n} \frac{E_{c i}}{n} \ldots
$$

Where $E_{c i}$ is the consumed energy of the $\mathrm{i}$-th node and $\mathrm{n}$ represents the whole node of wireless sensor networks.

4) Packet delivery ratio: Refers to the rate of correct packets being delivered

$$
\operatorname{PDR}=\left(\frac{\mathrm{z}}{\mathrm{m}}\right) * 100
$$

Table 2 provides percentage enhancement regarding HDN for both proposed protocols MPEGASIS and MPEGASIS with ALO and with other protocols for the scenario (BS being at middle). The use of HDN indicates that considerable amount of attention is given as the approximated value for the average of both lifetime and performance of the network. Hence, Table 2 only focuses on HDN aspects. Ideally, comparing the percentage enhancement for the proposed MPEGASIS with ALO and MPEGASIS without both scenarios is necessary. In Table 3, the dead percentage of the presented work are sourced.

As can be viewed from Table 2, the amount of improvement for each case in MPECASIS-ALO, where HND has improved in MPECASIS-ALO over MPECASIS without the use of ALO by 5.20\%. In terms of Networks Lifetime, the enhancement was by an estimate of $8.66 \%$. Moreover, the Throughput enhancement was about $4.80 \%$ in comparison to End To End Delay enhancement which was about 5.29\%. Overall, the Avg. Consumed (remained) enhancement was about 3.60\% where the Energy End to End Delay enhancement resulted in $3.60 \%$.

For this study, any impact of scalability imposed on MPEGASIS and MPEGASIS with ALO is analyzed reliably. This study has emphasized large-scale networks. Therefore, only WSN-2 model is fully considered. In terms of simulation results, Table 3 presents the findings where it outlines the number of rounds observed for $1 \%, 25 \%, 50 \%$ and $70 \%$ of node death. It is observed that altering the number of the diffused nodes has no impact on the network's lifetime which is desirable.

Table 2. Percentage enhancement for MPECASIS-ALO

\begin{tabular}{ccc}
\hline Metric & MPECASIS-ALO & MPECASIS \\
\hline HNDs & $65.30 \%$ & $60.10 \%$ \\
Networks Lifetime & $59.86 \%$ & $51.20 \%$ \\
Throughput & $87.30 \%$ & $82.50 \%$ \\
End To End Delay & $29.20 \%$ & $23.91 \%$ \\
Avg. Consumed (remained) Energy & $44.10 \%$ & $40.50 \%$ \\
End To End Delay & $53.00 \%$ & $49.40 \%$ \\
\hline
\end{tabular}

Indonesian J Elec Eng \& Comp Sci, Vol. 23, No. 1, July 2021: 273 - 284 
Table 3. Scalability effect on the network lifetime (rounds) when BS at border

\begin{tabular}{cccccc}
\hline \multirow{2}{*}{ Protocol } & Number of SN1 & \multicolumn{4}{c}{ Percentage of Dead Node In Round1 } \\
& & $1 \%$ & $25 \%$ & $50 \%$ & $70 \%$ \\
\hline MPEGASIS & 200 & 429 & 630 & 695 & 745 \\
& 250 & 540 & 680 & 750 & 773 \\
\multirow{2}{*}{ MPEGASIS and ALO } & 300 & 541 & 719 & 786 & 816 \\
& 200 & 550 & 680 & 792 & 800 \\
& 250 & 555 & 740 & 790 & 830 \\
& 300 & 540 & 770 & 862 & 890 \\
\hline
\end{tabular}

\section{CONCLUSIONS}

This presented work has finalized that the novel technique used of joining MPEGASIS with ALO is ideal for reducing energy consumption occurring in nodes. This was accomplished using a number of stages. The first one was random distribution of nodes where clustering the map into circular regions is the next step. Then, all nodes were linked with the closest node in the region at which they were. After these stages are achieved, the Ant lion optimization was applied to improve the connection of nodes, as well gain the maximum life battery of the sensor. In terms of the MPECASIS-ALO amount of improvement for each case, HND development in MPECASIS-ALO over Original PECASIS was around 65\%. The Networks Lifetime development was approximately 59\%, Throughput development was about 87\%, End To End Delay development was an estimate of 29\%, Avg. Consumed (remained) development was $44 \%$ and finally the Energy End To End Delay development rounded up to $53 \%$. Overall, these findings indicate a longer lifetime for the node as well as a minimum cost.

\section{REFERENCES}

[1] A. H. S. Al-Safi, Z. I. R. Hani, and M. M. Abdul Zahra, "Using a hybrid algorithm and feature selection for network anomaly intrusion detection," J Mech Eng Res Dev, vol. 44, no. 4, pp. 253-262, 2021.

[2] M. Jabor, N. S. Radh, M. A. Al-kinani, and Z. S. Al-khafaji, "Optimization of Electro less of Nickel base coating for Cermet Cutting Tools Substrate," vol. 44, no. 3, pp. 30-40, 2021.

[3] A. J. Hussain, Z. S. Al-khafaji, and W. A. Hamza, "An Investigation into the Distribution of Internal Residual Stresses of Aluminum Plate Subjected to Thermal Load by Using Hole Drilling Technique," Journal of Mechanical Engineering Research and Developments, vol. 44, no. 5, pp. 402-411, 2021.

[4] D. Yi, H. Yang, "HEER-a delay-aware and energy-efficient routing protocol for wireless sensor networks," Comput. Netw, vol. 104, pp. 155-173, 2016, doi: 10.1016/j.comnet.2016.04.022.

[5] M. Sabet and H. Naji, "An energy efficient multi-level route-aware clustering algorithm for wireless sensor networks: a self-organized approach," Comput. Electr. Eng., vol. 56, pp. 399-417, 2016, doi: 10.1016/j.compeleceng.2016.07.009.

[6] G. Kannan, T. S. R. Raja, "Energy efficient distributed cluster head scheduling scheme for two tiered wireless sensor network," Egypt. Inform. J., vol. 16, pp. 167-174, 2015, doi: 10.1016/j.eij.2015.03.001.

[7] M. Aslam, E.U. Munir, M.M. Rafique, X. Hu, "Adaptive energy-efficient clustering path planning routing protocols for heterogeneous wireless sensor networks,” Sustain. Comput., Inform. Syst., vol. 12, pp. 57-71, 2016, doi: 10.1016/j.suscom.2016.09.001.

[8] Zidar, M, Georgilakis, P. S, Hatziargyriou, N. D, Capuder, T, Škrlec, D, "Review of energy storage allocation in power distribution networks: Applications, methods and future research," IET Gener. Transm. Distrib., vol. 3, pp. 645-652, 2016, doi: 10.1049/iet-gtd.2015.0447.

[9] W. Guo, W. Zhang, G. Lu, "PEGASIS protocol in wireless sensor network based on an improved ant colony algorithm," 2nd International Workshop on Education Technology and Computer Science ETCS 2010, pp. 64-67, 2010, doi: 10.1109/ETCS.2010.285.

[10] T. Li, F. Ruan, Z. Fan, J. Wang, and J.-U. Kim, "An improved PEGASIS routing protocol based on neural network and ant colony algorithm," Int. J. Future Gener. Commun. Networking, vol. 8, no. 6, pp. 149-160, 2015, doi: 10.14257/IJFGCN.2015.8.6.15.

[11] S. Ghosh, W. Bengal, "Enhanced PEGASIS using Ant Colony Optimization for data gathering in WSN," in: 2016 International Conference on Information Communication and Embedded Systems (ICICES), pp. 1-6, 2016, doi: 10.1109/ICICES.2016.7518930.

[12] S. Feng, B. Qi, L. Tang, "An improved energy-efficient PEGASIS-based protocol in wireless sensor networks," in: Proceedings - 2011 8th International Conference on Fuzzy Systems and Knowledge Discovery, FSKD 2011 4, pp. 2230-2233, 2011, doi: 10.1109/FSKD.2011.6020058.

[13] N. Ramluckun, V. Bassoo, "Energy-efficient chain-cluster based intelligent routing technique for Wireless Sensor Networks," Appl. Comput. Inf., vol. 16, no. 1/2, pp. 39-57, 2018, doi: 10.1016/j.aci.2018.02.004.

[14] S. Kohli, P. Pratim Bhattacharya, M. Kumar Jha, "Implementation of homogeneous leach protocol in three dimensional wireless sensor networks," Int. J. Sens. Wireless Commun. Control, vol. 6, no. 1, pp. 4-11, 2016.

[15] Rina Mahakud et al., "Energy Management in wireless sensor network using PEGASIS," Procedia Computer Science, vol. 92, pp. 207-212, 2016 Published by Elsevier B.V. This is an open access article under the CC BY-NCND license (http://creativecommons.org/licenses/by-nc-nd/4.0/). Peer-review under responsibility of the Organizing Committee of ICCC 201, doi: 10.1016/j.procs.2016.07.347. 
[16] Đoko Banđur, Branimir Jakšić, Miloš Banđur, and Srđan Jović, "An analysis of energy efficiency in Wireless Sensor Networks (WSNs) applied in smart agriculture," Received 5 November 2018; Received in revised form 25 November 2018; Accepted 9 December 2018,0168-1699/ (C) 2018 Elsevier B.V. Computers and Electronics in Agriculture, vol. 156, pp. 500-507, 2019, doi: 10.1016/j.compag.2018.12.016.

[17] Raj Priyadarshini R and Sivakumar N, "Minimizing transmission loss using inspired ant colony optimization and Markov Chain Monte Carlo in underwater WSN environment," Journal of Ocean Engineering and Science (2019), doi: 10.1016/j.joes.2019.05.007.

[18] Somauroo and V. Bassoo, "Energy-efficient genetic algorithm variants of PEGASIS for 3D Wireless Sensor Network," Applied Computing and Informatics, 2210-8327/ 2019 King Saud University. Production and hosting by Elsevier B.V. This is an open access article under the CC BY-NC-ND license, doi: 10.1016/j.aci.2019.07.002.

[19] K. Karunanithy and B. Velusamy, "Cluster-tree based energy efficient data gathering protocol for industrial automation using WSNs and IoT," Journal of Industrial Information Integration, vol. 19, 100156, 2020. Journal of Industrial Information Integration 19 (2020) 100156 Available online 12 June 2020 2452-414X/ () 2020 Elsevier Inc, doi: 10.1016/j.jii.2020.100156.

[20] Wendi Rabiner Heinzelman, Anantha Chandrakasan and Hari Balakrishnan, "Energy Efficient Communication Protocol for Wireless Microsensor Networks," Published in The Proceedings of the Hawaii International Conference On System Sciences, January 4-7, 2000, Maui, Hawaii, doi: 10.1109/HICSS.2000.926982.

[21] S. Lindsey and C. Raghavendra, "PEGASIS: Power-Efficient Gathering in Sensor Information Systems", Proceedings of the IEEE Aerospace Conference, vol. 3, pp. 1125-1130, Big Sky, MT, USA, March 2002, doi: 10.1109/AERO.2002.1035242.

[22] D. Wohwe Sambo, B. O. Yenke, A. Förster, P. Dayang, "Optimized clustering algorithms for large wireless sensor networks: A review," Sensors, vol. 19, no. 2, p. 322, 2019, doi: 10.3390/s19020322.

[23] N. Anjum, M. Ahmad and S. Ahmad, "Gateway based energy efficient routing: GEER," Int. J. Adv. Res., Ideas Innovations Technol., vol. 3, no.4, pp. 483-488, 2017, doi: 10.1109/BWCCA.2013.35.

[24] D. Sharma, A. Goap, A. K. Shukla, A. P. Bhondekar, in: Proceedings of 2nd International Conference on Communication, Computing and Networking, vol. 46, Springer Singapore, 2019, doi: 10.1007/978-981-13-1217-5, URL: http://link.springer.com/10.1007/978-981-13-1217-5

[25] Seyedali Mirjalili, "The Ant Lion Optimizer," Advances in Engineering Software, vol. 83, pp. 80-98, May 2015, doi: 10.1016/j.advengsoft.2015.01.010 0965-9978/ 2015 Elsevier Ltd. All rights reserved.

\section{BIOGRAPHIES OF AUTHORS}
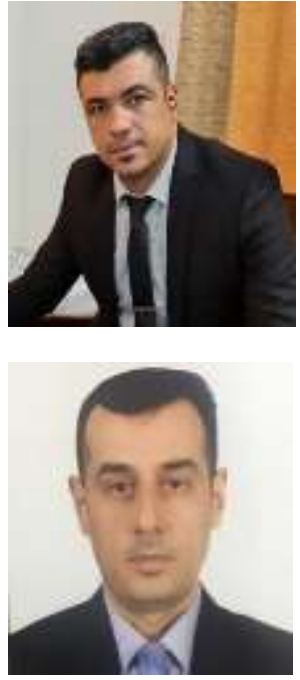

Ahmed Abdul Azeez Asmael, Middle Technical University, Technical Institute of Baqubah. A.A.Ismael worked and still in technical universities in Iraq since 2016 as a lecturer, The universities he worked for are Diyala University 2015, Al-Bayan Technical University 1016, Northern Technical University 2017, middle Technical University from 2019 to now. The author completed his MSC at Bangalore university, India 2014. He has experiences in the field of computer networks, the Internet and communications, where he worked at the Earth link company for the Internet and Communications for the years 2010 to 2012 as a maintenance engineer and programmer for communications devices. He has participated in international conferences, seminars and workshops in several technical fields. E-mail: Ahmed.abdulazeez@mtu.edu.iq

Basman Monther Al-Nedawe, Middle Technical University, Technical Institute of Baqubah. Basman Al-Nedawe is a Lecturer in the Technical institute of Baqubah at the Middle Technical University (Baghdad) where he has been a faculty member since 2004. He is the head of technical computer department since 2017. The author completed his Ph.D. at Huddersfield University (UK) and his undergraduate studies at Technology University (Iraq). His research interests lie in the area of communication systems, ranging from theory to design to implementation. He has collaborated actively with researchers in several other disciplines of electrical engineering, particularly error correction codes systems on problems at the hardware/software interface. The author has participated in many national and international scientific conferences, and he supervises an MSC program. Moreover, he has problem-solving skills, developed verbal and writing communication skills at University due to many reports and papers and presentations. He is a co-operative and capable of contributing positively while working as a part of the team due to group work at University also he is open minded to difference of opinions and negotiation skills. E-mail: b.al-nedawe@mtu.edu.iq 